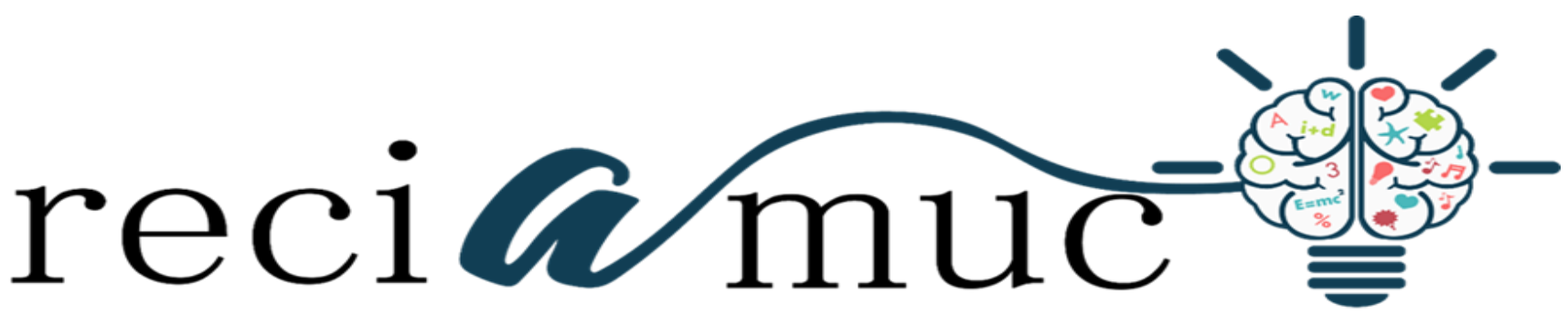

Revista cientifica de investigación actualización del mundo de las ciencias

Diana Karolina Mendoza Véliz ${ }^{\text {a; }}$ Karen Lissette Vallejo Gines ${ }^{\text {b; }}$ María José García Cedeño ${ }^{\text {c; }}$ Andrea Isabel Vallejo Maquilón d; Jhonny Xavier Zambrano Pico e; Bryan Anthony Guerra Jaime ${ }^{\mathrm{f}}$

Protección de los trabajadores que tienen riesgos en la exposición de gérmenes

Protection of workers who have risks in the exposure of germs

Revista Científica de Investigación actualización del mundo de las Ciencias. Vol. 3 núm., 1, enero, ISSN: 2588-0748, 2018, pp. 750-768

DOI: $10.26820 /$ reciamuc/3.(1).enero.2019.750-768

URL: http://reciamuc.com/index.php/RECIAMUC/article/view/256

Código UNESCO: 3205 Medicina Interna

Tipo de Investigación: Artículo de Revisión

Editorial Saberes del Conocimiento

Recibido: 10/12/2018

Aceptado: 20/01/2019

Publicado: 30/01/2019

Correspondencia: dianakarolinamendozav@gmail.com
a. Médico Cirujano; dianakarolinamendozav@gmail.com
b. Médico Cirujano; karenvallejog_22@hotmail.com
c. Médico Cirujano; dra.mariajosegc@ @otmail.com
d. Médico Cirujano; andreavallejo_90@hotmail.com
e. Médico; jhonnyxa14@hotmail.com
f. Interno de Medicina; bryanjaime@ hotmail.es 


\section{Protección de los trabajadores que tienen riesgos en la exposición de gérmenes}

Vol. 3, núm. 1., (2019)

Diana Karolina Mendoza Véliz; Karen Lissette Vallejo Gines; María José García Cedeño; Andrea Isabel Vallejo Maquilón; Jhonny Xavier Zambrano Pico; Bryan Anthony Guerra Jaime

\section{RESUMEN}

Todas las profesiones llevan implícito un riesgo inherente a la naturaleza misma de la especialidad y al ambiente donde se desenvuelve el trabajador. He allí, que los profesionales, pacientes y personal que labora en estos Centros Asistenciales de Salud al desarrollar sus actividades se encuentran expuestos a múltiples y variados riesgos laborales derivados del manejo de fluidos y/o secreciones biológicas, del medio ambiente físico, elementos químicos, ergonómico, y situaciones psicosociales que pueden repercutir no solo en la salud sino también en su desempeño profesional. Tal es así que los riesgos biológicos están presentes en todos los lugares del sector salud e incluyen gérmenes patógenos trasmitidos por aire y sangre, tales como los agentes causales de tuberculosis, hepatitis, infección por VIH/SIDA, entre otros. Corresponde entonces entender que, la exposición a los agentes biológicos, representa uno de los mayores riesgos a los que se enfrentan los profesionales de la salud, quienes para su seguridad necesitan cumplir una serie de normas, las cuales les permitirán estar libre de todo riesgo a accidente laboral, como a riesgo biológico y químico al que pueda ser accesible de no desarrollar este tipo de reglas sanitarias. Entiéndase con esto que, que es necesario velar porque estas unidades de atención médica se adapten y desarrollen de manera apropiada las normas de bioseguridad; las cuales son definidas como una doctrina de comportamiento encaminada a lograr actitudes y conductas que disminuyan el riesgo del trabajador de la salud de adquirir infecciones en el medio laboral. Esta normativa, compromete también a todas aquellas otras personas que se encuentran en el ambiente asistencial, medio éste, que debe estar diseñado en el marco de una estrategia de disminución de riesgos.

Palabras Claves: Riesgos Laborales; Exposiciones Bacteriológicas; Normas de Bioseguridad; Agentes Biológicos; Medio Laboral. 


\title{
Protección de los trabajadores que tienen riesgos en la exposición de gérmenes
}

Vol. 3, núm. 1., (2019)

Diana Karolina Mendoza Véliz; Karen Lissette Vallejo Gines; María José García Cedeño;

Andrea Isabel Vallejo Maquilón; Jhonny Xavier Zambrano Pico; Bryan Anthony Guerra Jaime

\begin{abstract}
All professions imply an inherent risk to the very nature of the specialty and to the environment where the worker develops. There, the professionals, patients and staff that works in these Assistance Centers when developing their activities are exposed to multiple and varied occupational risks derived from the handling of fluids and / or biological secretions, from the physical environment, chemical elements, ergonomic, and psychosocial situations that can affect not only health but also their professional performance. This is so that biological risks are present in all parts of the health sector and include pathogens transmitted by air and blood, such as the causative agents of tuberculosis, hepatitis, HIV / AIDS infection, among others. It is therefore necessary to understand that exposure to biological agents represents one of the greatest risks faced by health professionals, who for their safety need to comply with a series of regulations, which will allow them to be free of all risks to Occupational accident, as a biological and chemical risk to which it may be accessible not to develop this type of sanitary rules. Understand with this that, it is necessary to ensure that these health care units adapt and develop in an appropriate manner the biosafety norms; which are defined as a doctrine of behavior aimed at achieving attitudes and behaviors that reduce the risk of the health worker to acquire infections in the workplace. This regulation also commits all those other people in the healthcare environment, half of which must be designed within the framework of a risk reduction strategy.
\end{abstract}

Key Words: Occupational Hazards; Bacteriological Exposures; Biosafety Norms; Biological Agents; Working Environment. 


\section{Protección de los trabajadores que tienen riesgos en la exposición de gérmenes}

Vol. 3, núm. 1., (2019)

Diana Karolina Mendoza Véliz; Karen Lissette Vallejo Gines; María José García Cedeño; Andrea Isabel Vallejo Maquilón; Jhonny Xavier Zambrano Pico; Bryan Anthony Guerra Jaime

\section{Introducción.}

La salud debe ser considerada a nivel mundial, como la base fundamental para el desarrollo de la humanidad, denominación ésta de gran relevancia, pues lleva a considerar la importancia comprendida en el desarrollo de programas de intención médica y de participación activa que se encuentren dirigidos hacia el bien social, específicamente aquellos dirigidos a la disminución y prevención de riesgos y enfermedades que afecten al sector laboral y ciudadanía en general, como es el caso de las enfermedades adquiridas a nivel intrahospitalario. Para (Camacho, 2012):

Las infecciones intra hospitalarias son un conjunto de enfermedades cuyo denominador común es el haber sido adquiridas en un Hospital o Centro Asistencial Ambulatorio, las mismas son causadas por gérmenes patógenos que requieren para su ubicación de tres elementos fundamentales; una fuente constituida por pacientes, personal de la institución, visitantes, que pueden tener una enfermedad aguda, estar en el periodo de incubación, sin enfermedad o portadora de un agente infeccioso donde se incluyen objetos del ambiente que pueden estar contaminados. Un huésped susceptible y una persona colonizada o infectada por un microorganismo y las gotas generadas por la persona, fuente principalmente durante la tos, estornudos, al hablar o microorganismos que permanecen suspendidos en el aire por largos periodos o partículas de polvo que contienen el agente infeccioso. (p.198).

El señalamiento descrito, lleva a interpretar la importancia que tienen los programas dirigidos a la aplicación de medidas de bioseguridad, higiene y salud laboral; por ser éstas las encargadas de prevenir enfermedades ocasionadas por infecciones intra - hospitalarias que generalmente son ocasionadas por accidentes, o, por el tipo de actividad que realizan los trabajadores de la salud en cada una de las áreas de servicio a la cual esté designado según su especialidad médica. En base a esto, (Gamarra, 2012), expone "los riesgos de salud constituyen un tema que cada vez cobra mayor importancia, desde el punto de vista laboral". (p.43). 


\section{Protección de los trabajadores que tienen riesgos en la exposición de gérmenes}

Vol. 3, núm. 1., (2019)

Diana Karolina Mendoza Véliz; Karen Lissette Vallejo Gines; María José García Cedeño; Andrea Isabel Vallejo Maquilón; Jhonny Xavier Zambrano Pico; Bryan Anthony Guerra Jaime

El planteamiento expuesto permite señalar que actualmente el tema de los riesgos en la exposición de gérmenes comprendidos en el sector salud son cada vez más preocupantes; por tal razón es necesario que estos Centros de Asistencia lleven a cabo las debidas intervenciones y seguimientos en lo que se refiere al cumplimiento preventivo de accidentes biológicos y laborales, es decir, todo Hospital, Clínica o Centro de Asistencia a la Salud debe proporcionarle a los profesionales de medicina, pacientes y trabajadores en general, los recursos establecidos para su seguridad, tales como equipos e insumos, destacándose entre los mismos: materiales para esterilización y desinfección de los instrumentos de trabajo, químicos - reactivos y material de uso preventivo en general, con los cuales se pueden obtener condiciones de seguridad adecuada.

En unión a lo expuesto, (Sierralta, 2012), sostiene "existen convenios de salud basados en patrones de precaución universal, los cuales se basan en el manejo correcto de todo material con riesgos de infección, procedimientos, entrenamientos, eliminación y otras acciones diseñadas para prevenir las infecciones laborales". (p.32). Por tal razón, es necesario que los Centros de Salud desarrollen políticas y medidas ocupacionales encargadas de dar cumplimiento a las correspondientes normas de bioseguridad, ya que de esa forma se estará garantizando la seguridad del profesional y demás trabajador de la salud, así como de sus pacientes.

Por consiguiente, es necesario comprender que las medidas preventivas o normas de bioseguridad son necesarias en todo centro de salud y las áreas de atención al paciente que las conforman, las cuales son adoptadas con el fin de evitar o disminuir los riesgos de adquirir agentes patógenos o sufrir cualquier accidente laboral que pueda generar el contagio de determinadas enfermedades. En esta línea de ideas, (Gozaine, 2013), refiere "las normas de bioseguridad son medidas preventivas que se implementan en las instituciones para proteger la salud y disminuir el riesgo de transmisión de microorganismos, los cuales están presentes en los ambientes que conforman las áreas hospitalarias”. (p.54).

Corresponde entonces señalar que, las normas de bioseguridad son aquellas que representan las medidas y disposiciones que buscan proteger la vida del profesional, paciente y trabajador de la salud, a través del manejo, actitud y conocimientos adecuados. De allí, que es necesario que todo el que labora en estas instituciones y sus respectivas áreas de servicio tengan el conocimiento en 


\section{Protección de los trabajadores que tienen riesgos en la exposición de gérmenes}

Vol. 3, núm. 1., (2019)

Diana Karolina Mendoza Véliz; Karen Lissette Vallejo Gines; María José García Cedeño; Andrea Isabel Vallejo Maquilón; Jhonny Xavier Zambrano Pico; Bryan Anthony Guerra Jaime

Bioseguridad hospitalaria correspondiente; pues son estas normativas, las que le permitirán a los trabajadores de los hospitales, pacientes y en especial a los médicos tratantes exponerse de manera inadecuada a los agentes infecciosos que los llevarían a sufrir riesgos de accidentes biológicos o laborales.

No obstante, se observa con preocupación que en algunos Centros de Salud, se han presentado accidentes laborales, los cuales en su efecto, se han generado por el desconocimiento que mantienen ciertos profesionales y trabajadores del área de la medicina hacia las medidas de bioseguridad o, en otros casos, a la falta de los insumos correspondientes para el cumplimiento de estas normas de bioseguridad, que en determinados casos presentan algunas de estas instituciones; planteamientos éstos que llevan a establecer la necesidad de interpretar la importancia que tiene la protección de los trabajadores en situación de riesgo biológico, lo cual lleva a los mismos, a exponerse de forma continua a los riesgos biológicos generados al momento de asistir a las pacientes.

\section{Tipo de Investigación.}

Dentro de toda práctica investigativa, se precisan acciones de carácter metodológico mediante las cuales, se logra conocer y proyectar los eventos posibles que la determinan, así como las características que hacen del acto científico un proceso interactivo ajustado a una realidad posible de ser interpretada. En este sentido, se puede decir, que la presente investigación corresponde al tipo documental, definido por (Dávila, 2012), "se ocupa del estudio de problemas planteados a nivel teórico, la información requerida para abordarlos se encuentra básicamente en materiales impresos, audiovisuales y /o electrónicos”. (p.41).

En consideración a esta definición, la orientación metodológica permitió la oportunidad de cumplir con una serie de actividades inherentes a la revisión y lectura de diversos documentos donde se encontraron ideas explicitas relacionadas con los tópicos encargados de identificar a cada característica insertada en el estudio. Por lo tanto, se realizaron continuas interpretaciones con el claro propósito de revisar aquellas apreciaciones o investigaciones propuestas por 


\section{Protección de los trabajadores que tienen riesgos en la exposición de gérmenes}

Vol. 3, núm. 1., (2019)

Diana Karolina Mendoza Véliz; Karen Lissette Vallejo Gines; María José García Cedeño;

Andrea Isabel Vallejo Maquilón; Jhonny Xavier Zambrano Pico; Bryan Anthony Guerra Jaime

diferentes investigadores, para luego dar la respectiva argumentación a los planteamientos, en función a las necesidades encontradas en la indagación.

\section{Fuentes Documentales.}

El análisis correspondiente a las características que predomina en el tema seleccionado, llevan a incluir diferentes fuentes documentales encargadas de darle el respectivo apoyo y en ese sentido cumplir con la valoración de los hechos a fin de generar nuevos criterios que sirven de referencia a otros procesos investigativos. Para (Arias, 2010), las fuentes documentales incorporadas en la investigación documental o bibliográfica, "representa la suma de materiales sistemáticos que son revisados en forma rigurosa y profunda para llegar a un análisis del fenómeno". (p.41). Por lo tanto, se procedió a cumplir con la realización de una lectura previa determinada por encontrar aquellos aspectos estrechamente vinculados con la protección de los trabajadores que tienen riesgos en la exposición de gérmenes", para luego explicar mediante un desarrollo las respectivas apreciaciones generales de importancia.

\section{Técnicas para la Recolección de la Información}

La conducción de la investigación para ser realizada en función a las particularidades que determinan a los estudios documentales, tiene como fin el desarrollo de un conjunto de acciones encargadas de llevar a la selección de técnicas estrechamente vinculadas con las características del estudio. En tal sentido, (Arias Ob cit) refiere, que es "una técnica particular para aportar ayuda a los procedimientos de selección de las ideas primarias y secundarias”. (p. 71).

Por ello, se procedió a la utilización del subrayado, resúmenes, fichaje, como parte básica para la revisión y selección de los documentos que presentan el contenido teórico. Es decir, que mediante su aplicación de estas técnicas se pudo llegar a recoger informaciones en cuanto a la revisión bibliográfica de los diversos elementos encargados de orientar el proceso de investigación. Tal como lo expresa, (Arias Ob cit) "las técnicas documentales proporcionan las herramientas esenciales y determinantes para responder a los objetivos formulados y llegar a resultados efectivos" (p. 58). Es decir, para responder con eficiencia a las necesidades investigativas, se introdujeron como técnica de recolección el método inductivo, que hizo posible 


\section{Protección de los trabajadores que tienen riesgos en la exposición de gérmenes}

Vol. 3, núm. 1., (2019)

Diana Karolina Mendoza Véliz; Karen Lissette Vallejo Gines; María José García Cedeño; Andrea Isabel Vallejo Maquilón; Jhonny Xavier Zambrano Pico; Bryan Anthony Guerra Jaime

llevar a cabo una valoración de los hechos de forma particular para llegar a la explicación desde una visión general.

Asimismo, se emplearon las técnicas de análisis de información para la realización de la investigación que fue ejecutada bajo la dinámica de aplicar diversos elementos encargados de determinar el camino a recorrer por el estudio, según, (Arias, Ob cit) las técnicas de procesamiento de datos en los estudios documentales "son las encargadas de ofrecer al investigador la visión o pasos que debe cumplir durante su ejercicio, cada una de ellas debe estar en correspondencia con el nivel a emplear" (p. 123). Esto indica, que, para llevar a cabo el procesamiento de los datos obtenidos, es necesario establecer las técnicas que serán seleccionadas, destacándose en este caso, de manera particular: fichas de resumen, textual, registros descriptivos entre otros, los mismos se deben ajustar al nivel que ha sido seleccionado.

\section{Resultados.}

\section{Factores de Riesgo Biológico.}

Hablar de factores de riesgo biológico es hacer referencia al peligro existente dentro de los Centros de Salud de que las personas, sean éstas trabajadores o pacientes en general de adquirir determinada enfermedad, la cual puede ser transmitida, en alguno de los casos por la ausencia o fallas ante el debido uso de las normas de bioseguridad al momento de realizar la práctica médica, como por ejemplo los accidentes ocurridos por exposición a sangre o fluidos.

Entiéndase por riesgo biológico la posibilidad de adquirir enfermedad por el contacto con microorganismos reconocidamente patógenos, potencialmente patógenos o aquellos residuos contaminados con materia orgánica. Los contaminantes biológicos los podemos clasificar de dos tipos: Organismos vivos: bacterias, protozoos, virus, hongos, parásitos; y los derivados animales y vegetales: excrementos, restos cutáneos como pelos y plumas, sustancias antigénicas como enzimas y proteínas; polen, polvo de madera, esporas fúngicas, etc.

En virtud de lo mencionado se debe resaltar que, la principal vía de absorción de los contaminantes biológicos es la vía dérmica, bien por contacto directo por heridas o por 


\section{Protección de los trabajadores que tienen riesgos en la exposición de gérmenes}

Vol. 3, núm. 1., (2019)

Diana Karolina Mendoza Véliz; Karen Lissette Vallejo Gines; María José García Cedeño;

Andrea Isabel Vallejo Maquilón; Jhonny Xavier Zambrano Pico; Bryan Anthony Guerra Jaime

inoculación accidental; de allí, que el riesgo contraer la infección depende de la frecuencia de exposiciones percutáneas o mucosas a sangre u otros líquidos contaminados como consecuencia de las actividades laborales, ya que los accidentes de trabajo, pueden estar presente en cualquier área de trabajo debido a que es un suceso imprevisto y repentino que sobrevienen por causa o por ocasión del trabajo y que produce una lesión orgánica o perturbación funcional; en donde todo el personal debe estar preparado para este momento.

Según (Lara, 2012), "se denomina AES, a todo contacto con sangre o fluidos corporales y que lleva una solución de continuidad (pinchazo o herida cortante) o con contacto con mucosa o piel lesionada (eczema, excoriación, etc.)".(p.87). Lo expuesto por el autor lleva a considerar los accidentes por exposición a sangre o fluidos como una de las posibles causas de accidentes laborales que pueden ocurrir dentro de los Centros de Asistencia de Salud, los cuales en acompañamiento de virus de alto riesgo y otro tipo de factores ponen en riesgo la salud de los trabajadores de la Salud e incluso de sus pacientes.

Entre los agentes infecciosos transmitidos por accidentes por exposición a sangre o fluidos se encuentran los denominados "fuente", los cuales pueden ser transmitidos en el curso de un accidente. De allí, que los agentes frecuentemente más comprometidos son:

Virus de Inmunodeficiencia Humana (VIH): el riesgo de infectarse por este virus en un accidente laboral a través de una aguja que tiene sangre contaminada es estimado en 0.5 - $1 \%$. En un contacto mucoso con sangre contaminada baja a un $0.05 \%$.

Hepatitis A Virus $\boldsymbol{B}(\boldsymbol{H B})$ : el riesgo de infectarse por este virus en un accidente laboral a través de una aguja que tiene sangre contaminada es promedio un $15 \%$, llegando hasta un $40 \%$.

Hepatitis $\boldsymbol{A}$ Virus $\boldsymbol{C}(\boldsymbol{H V C})$ : el riesgo en este caso no está todavía bien precisado citándose cifras de hasta un $10 \%$.

Riesgo Laboral.

Representan toda posibilidad de que un trabajador sufra un determinado daño a su salud, como consecuencia del trabajo realizado. Cuando esta posibilidad se materialice en un futuro 


\section{Protección de los trabajadores que tienen riesgos en la exposición de gérmenes}

Vol. 3, núm. 1., (2019)

Diana Karolina Mendoza Véliz; Karen Lissette Vallejo Gines; María José García Cedeño; Andrea Isabel Vallejo Maquilón; Jhonny Xavier Zambrano Pico; Bryan Anthony Guerra Jaime

inmediato y suponga un daño grave para la salud de los trabajadores, hablaremos de un riesgo grave e inminente. La materialización del riesgo laboral puede derivar en un daño a la salud del trabajador, que se puede manifestar mediante una enfermedad, una patología o una lesión.

Los factores de riesgo laboral provienen de las condiciones en las que se trabaja y dan lugar a diferentes tipos de accidentes, enfermedades profesionales y efectos para la salud, se clasifican en cuatro grupos:

- Factores de seguridad: se refiere a las condiciones materiales que influyen en los accidentes laborales como, por ejemplo, los pasillos y las superficies de tránsito, los equipos y los aparatos de elevación, vehículos de transporte, las maquinas, las herramientas, los espacios en que se trabaja, las instalaciones eléctricas, etc. Entre las consecuencias habituales que se producen por la existencia de este tipo de factores de riesgo, encontramos las lesiones del trabajador originadas por los elementos móviles de las maquinas, golpes, atrapamientos, cortes, caídas de materiales, lesiones por herramientas manuales o mecánicas, lesiones oculares, esguinces, aplastamientos, caídas, quemaduras, asfixia; paro 16 respiratorio, etc.

- Factores derivados de las características del trabajo: contemplan los esfuerzos, la participación de las cargas, las posturas de trabajo, los niveles de atención requerida, la carga mental, etc., asociados a cada tipo de actividad. Las consecuencias pueden ser: irritabilidad, falta de energía y vitalidad, depresión, dolores de cabeza, mareos, insomnio, problemas digestivos, etc.

- Factores derivados de la organización del trabajo Se incluyen las tareas que integran el trabajo, los trabajadores asignados a ellas, los horarios, las relaciones jerárquicas, la velocidad de ejecución, etc. Las consecuencias pueden ser: fatiga, insatisfacción, estrés, problemas psicológicos, etc.

- Factores de origen físico, químico o biológico: Los factores de origen físico hacen referencia a contaminantes físicos como el ruido, las vibraciones, la iluminación, la temperatura, la humedad, las radiaciones, etc. Los factores de origen químico son los que están presentes en el medio ambiente de trabajo en forma de gases, vapores, nieblas, aerosoles, humos, polvos, y que 


\section{Protección de los trabajadores que tienen riesgos en la exposición de gérmenes}

Vol. 3, núm. 1., (2019)

Diana Karolina Mendoza Véliz; Karen Lissette Vallejo Gines; María José García Cedeño; Andrea Isabel Vallejo Maquilón; Jhonny Xavier Zambrano Pico; Bryan Anthony Guerra Jaime

se combinan con el aire respirable. Los contaminantes biológicos están constituidos por bacterias, virus, hongos, protozoos, etc., causantes de las enfermedades profesionales. Las consecuencias de su existencia pueden ser sordera, aumento del ritmo cardiaco, quemaduras, hemorragias, cataratas.

\section{Prevención de Riesgos Laborales.}

El tema de seguridad en el trabajo se lleva a cabo bajo la acción de tres puntos importantes, para que ésta sea efectiva, como lo es la prevención propiamente dicha para eliminar o disminuir el riesgo, la formación en seguridad y la información sobre seguridad. Para (Garreto, 2013):

La meta de la seguridad y la salud es evitar lesiones y enfermedades relacionadas con el trabajo, y que, la empresa logrará esta meta mediante la educación de los trabajadores de los riesgos relacionados con su trabajo, instalando controles, definiendo los procedimientos laborales seguros y prescribiendo el equipo de protección personal adecuado. (p.88).

Lo anteriormente descrito, lleva a comprender que la seguridad y la salud laboral representan para cada organización un factor clave, representan un tema que basado en la disciplina que estudia los ambientes de trabajo con el objetivo de conservar y mejorar la salud de los trabajadores y la atención constante a las condiciones de trabajo para convertirlas en agradables y confortables, es una premisa que contribuye a conformar el escenario para que el hombre pueda trabajar y constituye uno de los elementos que influye en el desempeño de los trabajadores.

Por ende, la prevención de accidentes, el cumplimento del programa de seguridad y salud laboral y la concientización en esta temática, es responsabilidad de todos y cada uno de los integrantes de una organización, ya que cada uno debe prevenir accidentes notificar las condiciones de riesgo que puedan afectar el desempeño de las labores, así como la salud de los trabajadores o de todo el que esté presente en la empresa.

Para (Liendo, 2013), "la seguridad laboral representa el cumplimiento de un conjunto de normas previamente pre establecidas que tienen como fin garantizar la seguridad del trabajador". (p.88). 


\section{Protección de los trabajadores que tienen riesgos en la exposición de gérmenes}

Vol. 3, núm. 1., (2019)

Diana Karolina Mendoza Véliz; Karen Lissette Vallejo Gines; María José García Cedeño; Andrea Isabel Vallejo Maquilón; Jhonny Xavier Zambrano Pico; Bryan Anthony Guerra Jaime

He ahí, la importancia del cumplimento de este tipo de normativas, las cuales en su efecto deben ser establecidas por cada gerencia como una política obligatoria a seguirse dentro de cada organización.

\section{Bioseguridad.}

La bioseguridad es conceptualizada como el conjunto de medidas preventivas que tienen como objeto proteger la salud y seguridad personal de los profesionales de salud y pacientes frente a los diferentes riesgos producidos por agentes biológicos, físicos, químicos y mecánicos. Dichas normas indican cómo hacer para cometer menos errores y sufrir pocos accidentes y, si ellos ocurren, cómo debemos minimizar sus consecuencias (OMS, 2012), concepto del que se comprende como una doctrina de comportamiento encaminada a lograr actitudes y conductas que disminuyan el riesgo del trabajador de salud de adquirir infecciones en el medio laboral, compromete también a todas aquellas personas que se encuentran en el ambiente asistencial, ambiente que debe estar diseñado en el marco de una estrategia de disminución de riesgos.

\section{Imagen $\mathbf{N}^{\circ}$ 1. Normas De Bioseguridad Médica.}

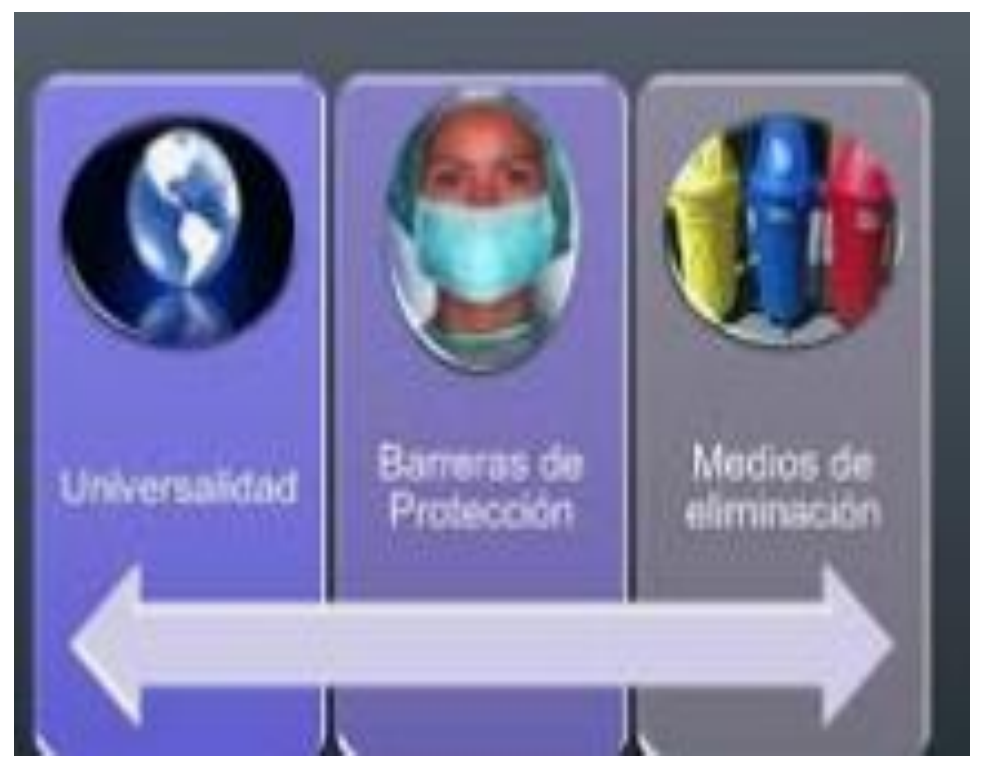

Fuente: Organización Mundial de la Salud (2012) 


\section{Protección de los trabajadores que tienen riesgos en la exposición de gérmenes}

Vol. 3, núm. 1., (2019)

Diana Karolina Mendoza Véliz; Karen Lissette Vallejo Gines; María José García Cedeño; Andrea Isabel Vallejo Maquilón; Jhonny Xavier Zambrano Pico; Bryan Anthony Guerra Jaime

Por consiguiente, para evitar la propagación de las enfermedades o contagios debe interrumpir el proceso de transmisión de las mismas. De allí que se hace necesario tomar medidas protectoras para todas las personas que están en un mismo ambiente. Durante el trabajo es esencial tener en cuenta los principios básicos de bioseguridad, en todos los ambientes de salud incluyendo los espacios que comprenden los laboratorios clínicos, en los que tanto el profesional como el paciente y las personas que allí laboran están expuestas, a contagios de enfermedades debido al existente riesgo de factores biológicos a los que se encuentran expuestos.

\section{Medidas de Bioseguridad.}

El significado de la palabra bioseguridad se entiende por sus componentes: "bio" de bios (griego) que significa vida, y seguridad que se refiere a la calidad de ser seguro, libre de daño, riesgo o peligro. Por lo tanto, bioseguridad es la calidad de que la vida sea libre de daño, riesgo o peligro. Para (Treviño, 2012), la Bioseguridad es:

Un conjunto de normas o medidas preventivas que deben tomar el personal que trabaja en áreas de la salud, para evitar el contagio de enfermedades de los pacientes en el área hospitalaria y en el medio en general, por la exposición de agentes infecciosos. (p.17).

Corresponde entonces señalar que, la bioseguridad hospitalaria, vista, a través de medidas científicas organizativas, es la que define las condiciones con que los agentes infecciosos deberían ser manipulados para reducir la exposición del personal en las áreas hospitalarias críticas y no críticas, a los pacientes y familiares, y al material de desecho que contamina al medio ambiente. De ahí, que investigadores como Lara (2012), opinen que "las medidas de bioseguridad que se tomen serán más estrictas cuanto más peligrosos sean los gérmenes que se manejan en el área en el cual se trabaja.” (p.123). Es decir, las medidas de bioseguridad deben ser una práctica rutinaria en las unidades médicas, las mismas deberán ser cumplidas por todo el personal que labora en los centros, independientemente del grado de riesgo según su actividad y de las diferentes áreas que compone el hospital. 


\section{Protección de los trabajadores que tienen riesgos en la exposición de gérmenes}

Vol. 3, núm. 1., (2019)

Diana Karolina Mendoza Véliz; Karen Lissette Vallejo Gines; María José García Cedeño; Andrea Isabel Vallejo Maquilón; Jhonny Xavier Zambrano Pico; Bryan Anthony Guerra Jaime

\section{Principios de Bioseguridad:}

Son las pautas que rigen las leyes o normas de la bioseguridad entre ellas según la OMS (2012):

a) Universalidad: Implica considerar que toda persona puede estar infectada, Asimismo considerar todo fluido corporal como potencialmente contaminante. Las medidas deben involucrar a todos los pacientes de todos los servicios, independientemente de conocer o no su serología. Todo el personal debe seguir las precauciones estándares rutinariamente para prevenir la exposición de la piel y de las membranas mucosas, en todas las situaciones que puedan dar origen a accidentes, estando o no previsto el contacto con sangre o cualquier otro fluido corporal del paciente. Estas precauciones, deben ser aplicadas para todas las personas sin excepción ni distinción, independientemente de presentar o no patologías.

Es importante entonces entender que, las precauciones universales o de bioseguridad son particularmente relevantes en los procedimientos médicos realizados, pues éstos pueden involucrar sangre o fluidos que pueden estar contaminados. Tales recomendaciones para el control de infecciones son de vital importancia para el personal que trabaja en los laboratorios clínicos. De allí, que, a partir de 1996, se ha actualizado el protocolo para el control de infecciones y ha incluido un número mayor de precauciones universales para la prevención de transmisiones de patógenos que viven en la sangre, expandiendo así los principios de bioseguridad a todos los fluidos corporales para minimizar el riesgo de infección cruzada entre pacientes y trabajadores de la salud, estableciendo para ello:

b) Uso de barreras: Comprende el concepto de evitar la exposición directa a sangre y otros fluidos orgánicos potencialmente contaminantes, mediante la utilización de materiales adecuados que se interpongan al contacto de los mismos. La utilización de barreras (ej. guantes) no evitan los accidentes de exposición a estos fluidos, pero disminuyen las consecuencias de dicho accidente. 


\section{Protección de los trabajadores que tienen riesgos en la exposición de gérmenes}

Vol. 3, núm. 1., (2019)

Diana Karolina Mendoza Véliz; Karen Lissette Vallejo Gines; María José García Cedeño;

Andrea Isabel Vallejo Maquilón; Jhonny Xavier Zambrano Pico; Bryan Anthony Guerra Jaime

\section{Imagen $\mathbf{N}^{\circ}$ 2. Uso De Barreras.}

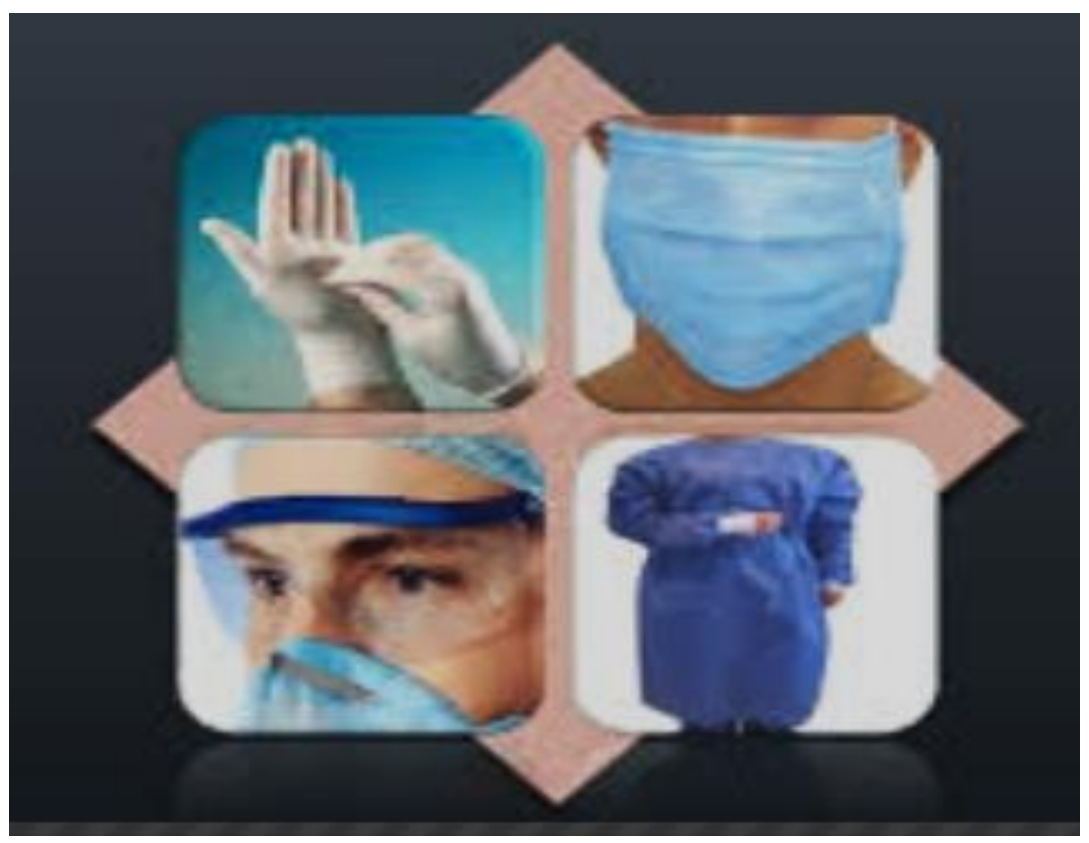

Fuente: Organización Mundial de la Salud OMS (2012)

c) Medios de eliminación de material contaminado: Comprende el conjunto de dispositivos y procedimientos adecuados a través de los cuales los materiales utilizados en la atención de pacientes, son depositados y eliminados sin riesgo de contagio por mal manejo. En atención a lo expuesto, cabe destacar que las medidas de bioseguridad y control de infecciones se basan en los siguientes principios.

- Inmunización del personal.

- Barreras protectoras.

- Lavado y cuidado de las manos.

- Utilización racional del instrumental punzante.

- Desinfección y esterilización del instrumental.

- Limpieza y desinfección de superficies contaminadas.

- Eliminación de desechos y material contaminado. 


\section{Protección de los trabajadores que tienen riesgos en la exposición de gérmenes}

Vol. 3, núm. 1., (2019)

Diana Karolina Mendoza Véliz; Karen Lissette Vallejo Gines; María José García Cedeño; Andrea Isabel Vallejo Maquilón; Jhonny Xavier Zambrano Pico; Bryan Anthony Guerra Jaime

Factores de Riesgo a los que se encuentran expuestos las personas (pacientes, trabajadores, y profesionales de la Salud) en los Centros de Asistencia Hospitalaria.

En toda Unidad de Atención de la Salud comprende entre sus funciones e instalaciones una serie de riesgos, los cuales deben ser abordados, a través de las diferentes normativas médicas conocidas como medidas de bioseguridad, a fin de prevenir los riesgos a sufrir de accidentes laborales y contagio de enfermedades infecciosas. Para (Rodríguez, 2012):

Los accidentes laborales, representan todos aquellos acontecimientos o sucesos producidos dentro del trabajo que se desarrollan en el cumplimiento de una función común o específica, debido a la exposición a uno o varios elementos que aumenta la probabilidad de que aparezca una enfermedad u otro resultado específico de daño a la salud. (p.76)

Lo expuesto por los autores permite interpretar que, los accidentes laborales representan las diferentes formas en que un profesional, en este caso, de la salud puede generar una situación en la que pone en riesgo su salud y la de los pacientes que acuden a estos Centros Hospitalarios; destacándose entre los mismos: los accidentes con elementos punzo cortantes, con fluidos corporales, los físicos, como caídas o traumas en el manejo de pacientes, la intoxicación química y los psicológicos. Estos últimos de vital importancia, puesto que generalmente ocurren debido a que el personal de salud se ve afectado de manera alarmante por situaciones que no le permiten realizar adecuadamente sus labores de una manera normal.

En unión de lo descrito, se señalan los riesgos biológicos, citados por (Leoni, 2012), como Riesgo "agentes vivos o inertes capaces de producir enfermedades infecciosas o reacciones alérgicas, producidas por el contacto directo de las personas que están laboralmente expuestas a estos agentes". (p.87). Destáquese entre éstos, los de origen percutáneos (pinchazos) o contacto con sangre o fluidos corporales, parenteral, secreciones infectantes y por vía respiratoria, siendo los principales agentes virales contaminantes del personal de enfermería son la hepatitis B y C, por VIH y por bacterias como la tuberculosis y el tétanos, entre otros. 


\section{Protección de los trabajadores que tienen riesgos en la exposición de gérmenes}

Vol. 3, núm. 1., (2019)

Diana Karolina Mendoza Véliz; Karen Lissette Vallejo Gines; María José García Cedeño;

Andrea Isabel Vallejo Maquilón; Jhonny Xavier Zambrano Pico; Bryan Anthony Guerra Jaime

En la misma línea de ideas, se presentan los riesgos ergonómicos, los cuales según Leoni (Ob cit), se refieren "a las características del ambiente de trabajo que causa un desequilibrio entre los requerimientos del desempeño y la capacidad de los trabajadores en la realización de las tareas". (p.90). Estos factores de riesgo están asociados con la postura, la fuerza, el movimiento, las herramientas, los medios de trabajo y el entorno laboral.

Con igual importancia, se citan los riesgos físicos, entendidos por Leoni (Ob cit), como "son distintas formas de energía, que, generados por fuentes concretas, pueden afectar a los trabajadores sometidas a ellas; estas energías pueden ser: Mecánicas, Térmicas, o Electromagnéticas". (p.43). Destáquese en este caso, los accidentes generados, ya sea, a consecuencia de fallas en equipos y ausencia de insumos.

Ya para finalizar, se citan los riesgos Químicos, entendidos por Casal (ob cit), como "aquellos constituidos por materia inerte (no viva). Pueden presentarse en el aire en forma de moléculas individuales (gas o vapor), o de grupos de moléculas unidas, formando aerosoles (sólidos o líquidos)”. (p.54). Es importante la diferencia entre ambos, porque los aerosoles, debido a su mayor tamaño, tienen un comportamiento en el aire y al ser inhalado, diferente del de los gases y vapores, que es idéntico al del aire por tratarse de moléculas individuales.

\section{Conclusiones.}

La salud, desde siempre ha representado el elemento humano más indispensable para alcanzar el desarrollo social de un país, bajo esa perspectiva las sociedades deben realizar acciones dirigidas a promover condiciones y espacios saludables. He aquí, que uno de los escenarios de la promoción de la salud es el escenario laboral, donde se deben aplicar estrategias y desarrollar acciones dirigidas a promover entornos saludables para las personas que trabajan; de allí, la importancia de la salud ocupacional, la cual debe ser concebida, a través de un concepto amplio y positivo de esta condición humana, pues implica defender y elevar la calidad de vida y la dignidad de la persona que trabaja.

En atención a esto, se presentan los Centros de Asistencia de Salud y los Laboratorios Clínicos, los cuales deben mantener una serie de normativas de prevención; las cuales les permitan 


\section{Protección de los trabajadores que tienen riesgos en la exposición de gérmenes}

Vol. 3, núm. 1., (2019)

Diana Karolina Mendoza Véliz; Karen Lissette Vallejo Gines; María José García Cedeño; Andrea Isabel Vallejo Maquilón; Jhonny Xavier Zambrano Pico; Bryan Anthony Guerra Jaime

minimizar el riesgo de contagio de bacterias y gérmenes existente en algunas enfermedades infecciosas y de accidentes laborales; por lo que deben estar guiados por la aplicación de medidas de bioseguridad como requisito sanitario necesario para el resguardo de la dignidad humana, la equidad, la solidaridad y la ética profesional; elementos necesarios para centrarse en las personas y de esa manera tener la salud como objetivo.

En atención a lo descrito, se de carácter obligatorio que en todo Centro de Salud sean aplicadas las normas de bioseguridad; estableciéndose con esto, que los lineamientos que comprenden esta filosofía, logren fomentar un servicio basado en la excelencia, previniendo de esa forma, cualquier tipo de riesgo y transmisión de enfermedad, de todo personal, paciente y profesional de la salud, sin excepción alguna, ya que de esta manera estos trabajadores estarán minimizando los riesgos a contraer cualquier tipo de infección ante la serie de microorganismos o bacterias a los cuales se encuentran expuestos, evitándose de esa forma el contagio de gérmenes e infecciones tales como, Síndrome de Inmuno Deficiencia Adquirida, Gripes, Hepatitis B, entre otras...,

En términos generales, es necesario el personal que labora en los diferentes Centros de Salud, exponiéndose a riesgos laborales y manipulando cualquier tipo de muestras biológicas cumpla con las normas de bioseguridad existentes, las cuales consisten en los principios, técnicas y prácticas que tienen como propósito disminuir el riesgo de transmisión de microorganismos de fuentes reconocidas o no reconocidas, de infección en servicios de salud vinculadas a accidentes por exposición a sangre y fluidos corporales que les generan a estos trabajadores una serie de consecuencias biológicas, e incluso en el peor de los casos hasta la muerte.

\section{Bibliografía.}

Arias, F. (2010). Paradigmas de la Investigación Científica. España: Editorial: Luces.

Camacho, P. (2012). Infecciones Intra - Hopsitalarias . Lima . Perú: [Tesis de postgrado]. Lima:.

Dávila, N. (2012). Paradigmas de la Investigación Científica. Pereire, Colombia: Editorial: Las Brisas. 


\section{Protección de los trabajadores que tienen riesgos en la exposición de gérmenes}

Vol. 3, núm. 1., (2019)

Diana Karolina Mendoza Véliz; Karen Lissette Vallejo Gines; María José García Cedeño;

Andrea Isabel Vallejo Maquilón; Jhonny Xavier Zambrano Pico; Bryan Anthony Guerra Jaime

Gamarra, V. (2012). Riesgos Ocupacionales y a la Salud del Trabajador en el campo Médico. España: Editorial: Elsevier.

Garreto, D. (2013). Riesgos Laborales en Contextos de Salud. Medellín: Cuarta Edición. Colombia: Corporación para Investigaciones Médicas.

Gozaine, J. (2013). Nor,as de Bioseguridad en Centros Hospitalarios. Oriente: Escuela de Medicina en la Universidad de Oriente. UDO.Venezuela.

Lara, G. (2012). Contaminación Biológica. Riesgo Ocupacional. México.: Editorial. Limusa. México.

Leoni, P. (2012). Riesgos Biológicos en Centros Hospitalarios. Revista Vida Preventova Journal Medic, 14.

Liendo, H. (2013). Seguridad Laboral. Normas de Bioseguridad. Madrid: Editorial: Espasa Calpe.

OMS, O. M. (2012). Normas de Bioseguridad en la prevención de Contagios. Revista Vida y Salud de la Universidad Nacional Autónoma de México, 14.

Rodríguez, J. (2012). Accidentes y Riesgos Ocupacionales en Salud. Chile: Tercera Edición. Santiago de Chile, Publicaciones Técnicas Mediterráneas.

Sierralta, R. (2012). Riesgos Ocupacionales a la Salud. Perú: Universidad Peruana Cayetano.

Treviño, G. (2012). Bioseguridad: Normas Hospitalarias. Revista Salud Integral de la Universidad de la Habana en Cuba, 17. 